\title{
The acoustic properties of capsaicin-induced cough in healthy subjects
}

\author{
M.J. Doherty*, L.J. Wang*, S. Donague*, M.G. Pearson*, \\ P. Downs**, S.A.T. Stoneman**, J.E. Earis*
}

The acoustic properties of capsaicin-induced cough in healthy subjects. M.J. Doherty, L.J. Wang, S. Donague, M.G. Pearson, P. Downs, S.A.T. Stoneman, J.E. Earis. CERS Journals Ltd 1997.

ABSTRACT: Acoustic analysis of cough both in the time and frequency domain has been reported using voluntary and spontaneous cough. The main aim of this study was to discover whether such analysis of capsaicin-induced cough enables differences between normal subjects to be recognized.

We present data from 13 healthy subjects (with normal lung function and no history of respiratory disease) using a new method of acoustic analysis, which presents the data in three graphical forms: 1) spectrogram; 2) overall spectral energy, 3) root mean square (RMS) pressure plots.

Using the RMS sound pressure traces, different subjects had either two peaks, a single peak or multiple peaks. The occurrence of single and multiple peaks has previously been associated with disease states but we found them in normal subjects. The number of peaks and the visual pattern of the spectrogram was reproducible within and specific to each individual over time. During a peal of coughs in a single expiration, the peak amplitude of successive coughs decreased as lung volume reduced. Despite similarities in the overall spectral energy between individuals, there were marked differences in the small visual details of the spectrograms. However, in an individual, these small details were remarkably constant both within and between days, and can be regarded as a "cough signature".

This type of spectrographic analysis provides a new approach to the analysis both of normal and abnormal cough sounds, and has identified similarities and differences in capsaicin-induced cough in normal individuals. It has potential as a tool with which to study the pathophysiology of cough.

Eur Respir J., 1997; 10: 202-207.
*Aintree Chest Centre, Fazakerley Hospital, Liverpool. **Dept of Civil Engineering, University College of Swansea, Wales.

Correspondence: J.E. Earis

Aintree Chest Centre

Fazakerley Hospital

Longmoor Lane

Liverpool L9 7AL

UK

Keywords: Capsaicin

cough

cough sounds

spectrogram

Received: January 111996

Accepted after revision September 101996
Cough is a powerful response to the stimulation of sensory nerves predominantly in the larynx, trachea and central airways. It is characterized by an initial contraction of the expiratory muscles against a closed glottis, followed by a violent expiration as the glottis opens suddenly [1]. In healthy subjects, cough is infrequent, is usually nonproductive, and is self-limiting. However, in patients with chronic chest disease, particularly when associated with cigarette smoking, cough is a very common and troublesome symptom [2, 3]. The sensitivity of the reflex can be tested by inhaling a tussigen. One such agent is the extract of red pepper, capsaicin, which when inhaled at low concentrations produces both doserelated and reproducible coughing [4].

Cough produces an easily recognizable sound, which can be recorded and used to count the number of coughs $[3,5]$. However, there has been little work published on the acoustic analysis of the cough sound [6,7]. In this study, we describe a method of analysing cough sounds using techniques usually employed for lung sound analysis [8], and apply this method of analysis to capsaicin-induced cough.

Previous studies have shown that cough in normal individuals can be distinguished from cough in disease states [6]. This suggests that there is considerable similarity between the acoustic features of cough in normals. Anecdotally, however, the human ear can sometimes distinguish between the cough of different normal subjects. The main aim of this study was to discover whether analysis of the time domain cough phase, overall spectral energy and the visual observation of the fine details of the cough spectrographs, enable differences in cough between normal subjects to be recognized.

\section{Methods}

\section{Subjects}

Thirteen healthy subjects ( 6 males and 7 females; median age 25 yrs, range 18-55 yrs) three of whom were current smokers were studied. Their median height was $173 \mathrm{~cm}$ (range 160-185 cm), median weight was $71 \mathrm{~kg}$ (range 51-89 kg), and no subject was obese (defined as a body mass index (BMI) of $>29 \mathrm{~kg} \cdot \mathrm{m}^{-2}$ ). Each subject had normal spirometry (mean forced expiratory volume in one second (FEV1) $111 \%$ of predicted (SD 15\%), 
forced vital capacity (FVC) $101 \%$ pred (SD 14\%)). The subjects had no respiratory symptoms or relevant past medical history or medication. No subject had suffered from a respiratory tract infection in the 4 weeks prior to the study, and clinical examination of the respiratory system revealed no abnormality in any of the subjects. All subjects gave informed consent and the study had approval from the Hospital Ethics Committee.

\section{Capsaicin cough test}

The capsaicin cough test was performed with a custombuilt dosimeter using the single inhalation technique. The dosimeter was designed to be activated by a small negative pressure, as during inhalation, with a time delay of $0.25 \mathrm{~s}$ and an inhalation time of $2 \mathrm{~s}$, during which period $0.009 \mathrm{~mL}$ of nebulized solution was delivered. The mass median diameter of nebulized particles was $5.2 \mu \mathrm{m}$, and inspiratory flow rate was governed by a fixed aperture to give a maximum flow rate of $0.75 \mathrm{~L} \cdot \mathrm{s}^{-1}$. The concentration of capsaicin was increased through eight doubling dilutions from 1.95 to $500 \mu \mathrm{M}$, spaced at 1 min intervals. The subjects inhaled the test solution from residual volume to total lung capacity. The concentration of capsaicin inducing five coughs (C5) [9] was repeated, and if found to be reproducible the test was terminated. Each subject underwent three capsaicin cough tests. On the first study day, two tests were performed (Series 1 and 2) with a gap of $30 \mathrm{~min}$ between the tests. On the second study day, 4 days after the first, a third test was performed (Series 3 ). In addition to the cough recordings, recordings of speech were also made using the same equipment and recording set-up. Each subjects speech was recorded by asking them to count audibly from 1 to 10 , to give an indication of the spectral characteristics of their speech. No further instructions were giving to the subjects as to how they should speak.

\section{Cough sound recording and analysis}

A unidirectional microphone (Shure SM58-CN; Evanston, IL, USA) with a flat frequency response from 50 $\mathrm{Hz}$ to $15 \mathrm{kHz}$ was set $225 \mathrm{~mm}$ from the subject, at an angle of $45^{\circ}$, both in the vertical and the horizontal plane to the axis of the cough. The signals were recorded on to a stereo cassette tape recorder (Yamaha KX650, Tokyo, Japan). The cough sounds were recorded in a quiet room and were approximately $40 \mathrm{~dB}$ above the level of the background noise.

The data were analysed by playing the recorded sounds back on a Yamaha KX650 stereo cassette tape recorder. A $10 \mathrm{~s}$ burst of sound was digitized at $20 \mathrm{kHz}$ by a Data Translation DT2831-G (Marlborough, MA, USA), 12bit analogue-to-digital conversion (ADC) board, mounted in a 486DX IBM compatible PC. The sampling was started manually by the operator triggering the ADC to capture the required part of the audio signal. The digitized data were stored on disk and processed with a custom-written software program to convert the data from the time to the frequency domain via a standard Fast Fourier Transformation (FFT) routine. Cough is a nonstationary signal (i.e. it varies continuously with time), and when conducting spectral analysis it is necessary to compare the time-dependent spectra on a single plot. This is achieved by plotting spectra alongside one another producing a three dimensional spectrogram; in this study a $\mathrm{Z}$ plot display was used [8]. To produce the $\mathrm{Z}$ plot, the data were processed in a series of blocks, each comprising 1,024 samples with an increment of 512 samples from the start of the preceding block; each $10 \mathrm{~s}$ record, therefore, produced 389 blocks which overlapped by $50 \%$ [10]. Each block was processed by the application of a Hanning window before conversion to the frequency domain by means of the FFT routine.

Figure 1 shows a typical printout of a cough analysis containing three areas of interest. Area A is a graph of

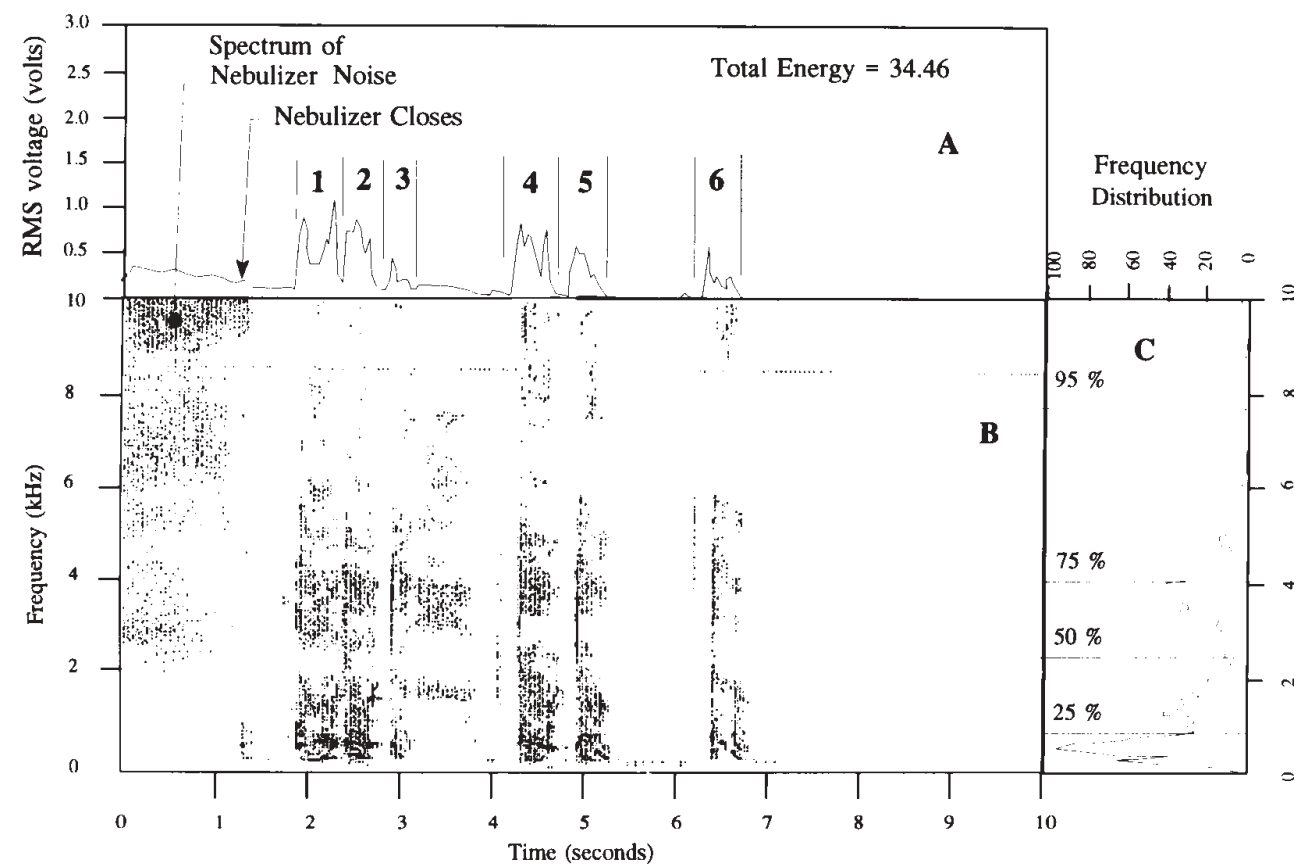

Fig. 1. - Printout of a typical analysis of cough. A: instantaneous root mean square (RMS) sound pressure level; B: spectrogram of frequency versus time with sound pressure level represented by a grey scale; C: spectral energy of that part of the spectrogram in which cough was present. The horizontal lines represent the frequencies below which lies the indicated percentage of the total energy of the spectrogram. 
the instantaneous root mean square (RMS) sound pressure level of cough. Considering the graph from time zero, it can be seen that inhalation terminates in a small localized peak due to the sound of the closure of the nebulizer valve at about $1.3 \mathrm{~s}$. There is then a quiet period, during which the subject continues to inhale until the onset of cough (defined as the "time to onset" of the first cough). The RMS trace then shows the individual coughs and where the peaks and troughs of energy within an individual cough lie, producing similar but more accurate and detailed information than the standard time domain tussigrams.

Area B is the spectrogram. Time is on the horizontal axis, frequency on the vertical axis and sound pressure level is represented by a grey scale. Moving from left to right, this shows the sound of the nebulizer lasting approximately $1.3 \mathrm{~s}$ followed immediately by a short low frequency sound, which represents the closure of the nebulizer valve. There is then a quiet period of about $0.6 \mathrm{~s}$, defined as the time to onset. There follows a series of three coughs, a short period of inspiration and a further two coughs. There is then a quiet pause and a final cough.

Area $\mathrm{C}$ is the spectral energy in that part of the spectrogram in which cough was present. It shows the frequency distribution of the acoustic energy in the coughs alone, with the spectral energy of the nebulizer having been excluded. The horizontal lines are the frequencies below which 25, 50, 75 (quartile frequencies) [7] and $95 \%$ (spectral edge frequency) of the total energy of the spectrogram is contained.

This form of data presentation yielded three methods to describe cough: 1) RMS plot; 2) spectrographic features; and 3) overall spectral energy.

\section{Results}

\section{RMS plot results}

The RMS plots (fig. 2) show three different types of energy pattern within individual coughs. The most common pattern, found in 8 of the 13 subjects, is a cough with two energy peaks (fig. 2a), one at the beginning and one at the end of the cough, separated by a low energy period. A single peaked cough sound was found in 3 of the 13 subjects (fig. 2 b) and three or more high energy peaks separated by low energy periods was observed in two subjects (fig. 2c). In individual subjects, these patterns were repeatable both within and between study days.

During a peal of coughs, that is a series of coughs within one expiration, the energy of each successive cough (peak amplitude) decreased in most of the plots. If at the end of such a peal of coughs the subject inspired before coughing again, then the subsequent RMS plot was of higher energy and the pattern was repeated.

\section{Spectrogram results}

There are a number of features which are used to judge visual similarity of spectral plots. These include the frequencies at which the dominant peaks of acoustic energy are located, the frequencies at which there is an absence of acoustic energy, and the number and timings of spectral events (e.g. the timings of cough events).
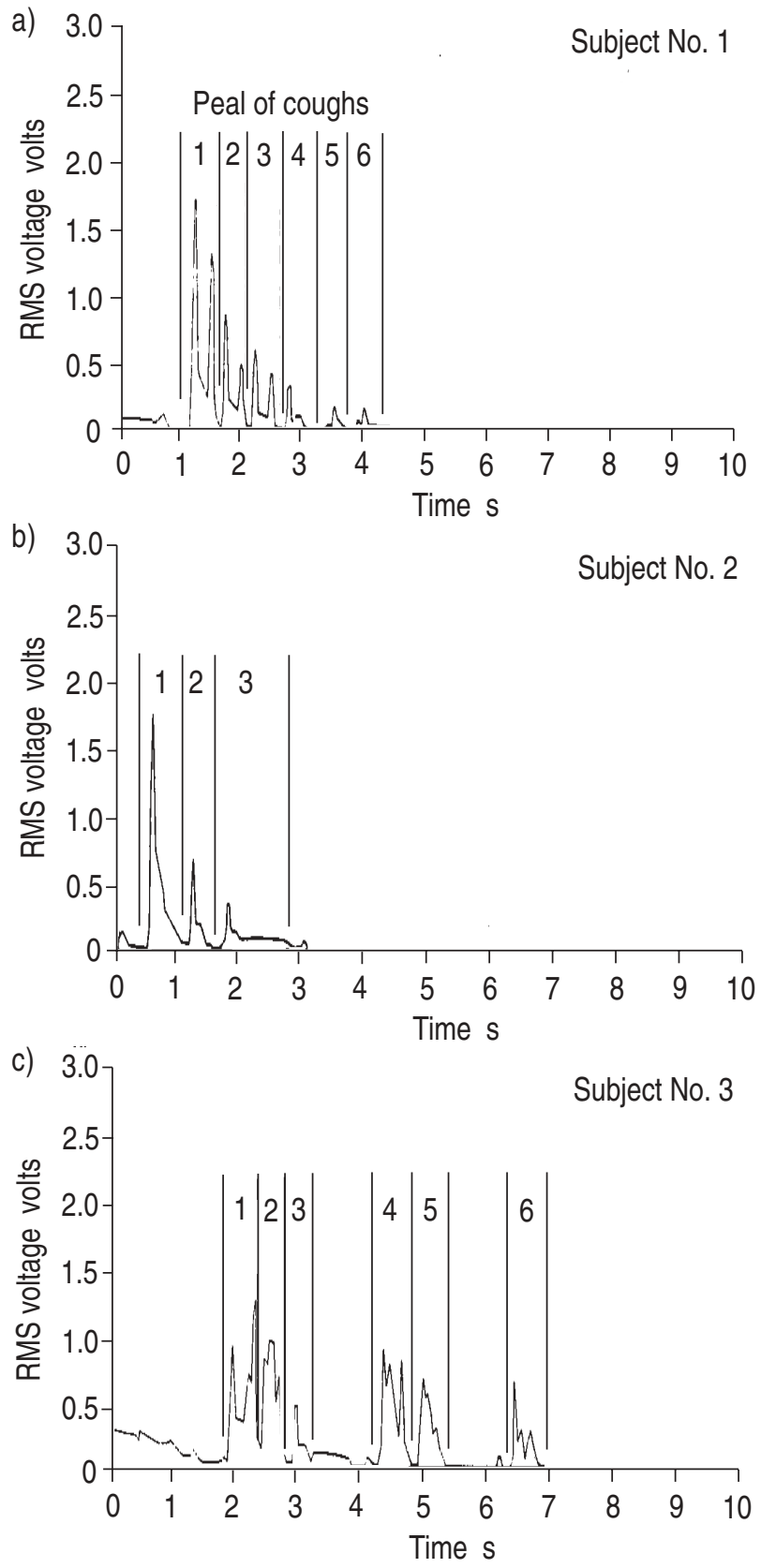

Fig. 2. - Instantaneous root mean square (RMS) plots showing three different patterns of individual coughs: a) double-peaked; b) singlepeaked; c) multiple-peaked.

The overall visual pattern of the spectra of an individual were similar between tests within a day and between tests on different days. However, spectrograms varied greatly between individuals. Figure 3 shows three spectra of induced cough sounds in the same individual, the first two recorded $30 \mathrm{~min}$ apart on the first day (fig. 3a and $3 \mathrm{~b}$, respectively), and the third recorded on the fourth day (fig. 3c). These spectrograms show great visual similarity; important features are indicating by the letters $\mathrm{A}, \mathrm{B}$ and $\mathrm{C}$. Area $\mathrm{A}$ is magnified in the box and shows similar spectral peaks at frequencies of $110,220,450$ and $825 \mathrm{~Hz}$ between tests. In addition, the frequency of particular events is reproducible, such as the $3,500 \mathrm{~Hz}$ maximum frequency of the final cough seen between 6 and $7 \mathrm{~s}$. Figure $4 \mathrm{a}$ is another spectrogram showing a 


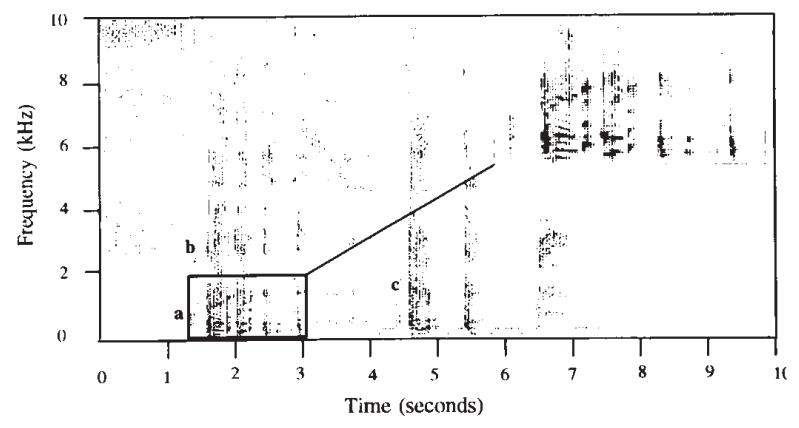

(a) Test 1 at Time 0

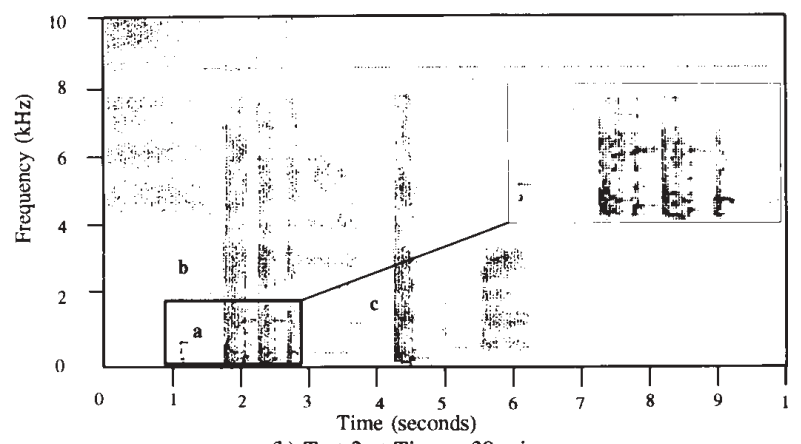

(b) Test 2 at Time +30 mins

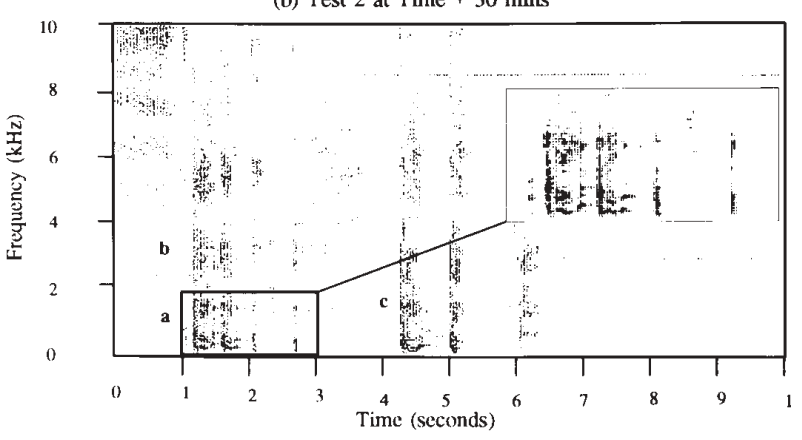

(c) Test 3 at Time +4 days

Fig. 3. - Three spectrographs from the same patient showing great visual similarity over time: Test 1 at Time 0 ; b) Test 2 at Time +30 min; and c) Test 3 at Time +4 days. Areas of similarity indicated by $\mathrm{a}, \mathrm{b}$ and $\mathrm{c}$.

peal of four coughs. Like the other spectra it manifests a series of time-varying spectral "structures". At approximately $250 \mathrm{~Hz}$, there is a band of acoustic energy ("r" shaped), which changes during the cough (shown magnified inset in the main figure). It first increases, peaks, and then decreases as the cough proceeds. Figure $4 \mathrm{~b}$ is the same patient recorded 4 days later and shows striking visual similarities.

In figure 3 , the cough referred to as "B" at $1.75 \mathrm{~s}$ and " $\mathrm{C}$ " at $4.25 \mathrm{~s}$ is characterized as much by the frequencies at which there is no acoustic energy as it is by the spectral peaks at which energy is present. The visual similarities between these areas of low spectral energy can be clearly seen by comparing the corresponding coughs during Tests 1,2 and 3. Figures $4 \mathrm{a}$ and $\mathrm{b}$ also show striking similarities between areas of low energy recorded on different test days.

\section{Spectral energy}

Significant acoustic spectral energy present up to 10 $\mathrm{kHz}$ and possibly beyond is clearly demonstrated in this
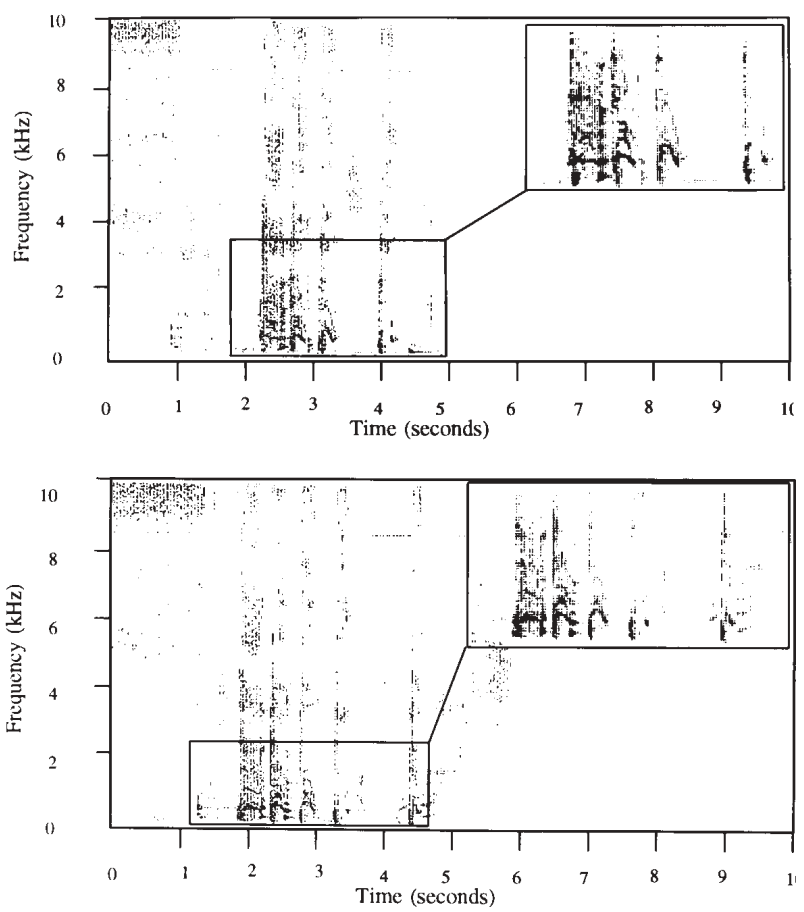

Fig. 4. - Two spectrographs from the same patient 4 days apart, showing similar visual features.

part of the analysis (fig. 1, Area C). In keeping with this observation the overall mean frequencies below which $25,50,75$ and 95 of the spectral energy of the 39 cough tracings (three results from each of the 13 patients) are relatively high as seen in figure 5 . The mean frequency at which $75 \%$ of the spectral energy occurred being $4.4 \mathrm{kHz}$.

The frequency below which $25 \%$ of the spectral energy occurred ranged $0.67-1.2 \mathrm{kHz}$, the $50 \%$ spectral energy level ranged $1.3-3.1 \mathrm{kHz}$, the $75 \%$ level $3.3-5.3 \mathrm{kHz}$, and the $95 \%$ level $6.5-8.7 \mathrm{kHz}$. Therefore, the distribution (maximum and minimum values) of the 25, 50, 75 and the $95 \%$ spectral energy levels did not overlap (fig. $5)$. This indicates that the relative energy distribution

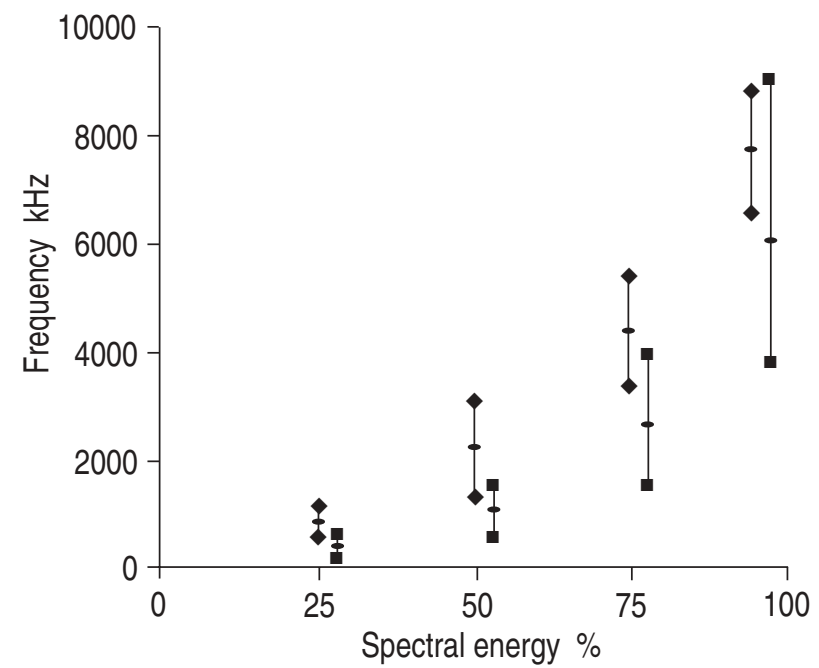

Fig. 5. - The group minimum, maximum and mean frequency level below which $25,50,75$ and $95 \%$ of the spectral energy occurred for cough $(-\rightarrow)$ and speech (- $\square$ ). For each of these results, all the data were used from each of the 13 patients. 
of coughs between subjects is similar, despite the fact that the visual characteristics of the spectrograms are quite different. In comparison, a similar analysis for speech on the same individuals is also seen in figure 5, and shows the $25 \%$ spectral energy level ranged $0.31-0.60 \mathrm{kHz}$, the $50 \%$ level ranged $0.60-1.5 \mathrm{kHz}$, the $75 \%$ level ranged $1.5-3.9 \mathrm{kHz}$ and the $95 \%$ level ranged $3.7-8.9 \mathrm{kHz}$. Thus, the frequency ranges of the 25, 50, 75 and $95 \%$ spectral energy levels for speech overlap.

\section{Discussion}

This study describes an experimental investigation of induced cough sounds in normal subjects. Previous studies have examined voluntary $[11,12]$ and spontaneous cough [7]. Induced cough has the advantage of stimulating a reflex, which is relatively independent of the action of the higher centres [13-16] and, thus, should result in more reproducible cough sounds. Capsaicin has been shown to produce reproducible, dose-related coughing [4] without tachyphylaxis [17], and little if any bronchospasm [4, 18]. The lack of induced bronchospasm is important, as it is to be expected that it will change the sound of the induced cough since the boundary geometry of the system generating the sounds will be changed.

Spectral frequencies and the pattern of the relative energy distribution were recorded in a reproducible manner. An external microphone has advantages over a chest wall microphone since the microphone frequency response is not altered by being attached to the skin, heart sounds are not recorded, tissue movement artefacts do not occur, and the effect of the chest wall as a low pass filter is obviated.

Consideration of time domain tussigrams have indicated the presence of two distinct peaks of energy in cough sounds of normal subjects. We believe the first sound follows glottic opening and that the second sound is associated with the closure of the glottis [19]. However, other studies have suggested that a closed glottis is not always necessary for cough production [20]. In this study using RMS sound pressure traces, 8 of the 13 subjects clearly showed two distinct energy peaks at the beginning and end of the cough. Other investigators [6, 11] have shown that some individuals do not have this double-peaked cough but instead have either a single energy peak or multiple peaks. These patterns are usually described in association with disease states, single cough sounds being associated with psychogenic cough or in patients with laryngectomies [6]. Multiple-peaked cough sounds were found in patients with chronic obstructive airways disease [6]. However, we found that single and multiple peaks occurred in normal subjects; in keeping with this observation is the recent report from THORPE et al. [21], who also showed that although single-peaked cough sounds were much commoner in disease states they occasionally occur in normals. These results suggest that the number of high energy peaks within a cough holds little specific diagnostic value, being a characteristic of the individual rather than of the disease.

The successive reduction of energy noted during a peal of coughs during a single expiration does not seem to have been noted previously, although it was shown in the figures that accompanied the paper by Young et al. [20]. The likely explanation for this observation is that the reduced pressure and flow rates at the lower lung volumes during a peal of coughs $[1,20]$ result in progressively less explosive glottic opening.

The overall energy content of cough in this study extended up to $10 \mathrm{kHz}$. This is significantly higher than that reported in previous studies, where energy between 2 to $2.5 \mathrm{kHz}$ was more common [12, 13]. However, PIIRILIA and SoviJarvi [7], recording cough at the manubrium sternum, found upper sound limits in the range 5.4-7 $\mathrm{kHz}$. Our findings are more consistent with the expected energy content of sounds heard at the mouth, and may represent the wider dynamic range of our instrumentation and use of an external microphone, thereby, avoiding attenuation due to chest wall effects.

When the whole energy spectrum of cough is analysed, there is a similarity of overall energy distribution between individuals, as exemplified by the quartile patterns (fig. $5)$. These similarities in energy distribution have been described previously [21], and suggest that cough sound production is very similar between subjects. Thus, this type of analysis can be used to compare normal and abnormal sounds. In contrast, the overlapping quartile energies in speech probably occur because the sound source at the vocal cords produces marked variation of pitch, even in an individual. Thus, it is likely that vocal cord vibration is not the predominant mechanism of generation of cough sounds; turbulent airflow and vibration of the airways themselves are the most dominant mechanisms. Despite these similarities in overall energy distribution, examination of the small visual features of the spectrograms showed marked differences between subjects. However, in an individual these features were remarkably constant both within and between days, and can be regarded as a "cough signature". This unique finding is in keeping with anecdotal observations that individuals can sometimes be recognized by their cough. Unfortunately, these visual similarities are not easily amenable to objective measurement or statistical analysis and are analogous to describing chest radiographic abnormalities; however, the use of advanced pattern recognition techniques may allow objective analysis. Although it is not possible to prove from the current study, it is, nevertheless, likely that the "cough signatures" in individuals are due to colouration of the underlying cough sound by the individual anatomical variations, particularly the various resonances within the upper airway, nasal spaces and sinus cavities. The powerful influences of the upper airway in modifying sound has been well demonstrated in cough [12] and speech research [22].

In conclusion, a new method of cough sound analysis has been described, which uses three different graphical forms to extract information from the cough sound signal. Using this method in the controlled situation of a capsaicin cough challenge, we have shown individual variations in spectrograms which are constant in an individual over time, despite the known similarities in overall sound pressure level distribution which exist between individuals. These small spectrographic features seen in normal individuals need further evaluation and quantification as they may help to improve the understanding of the underlying pathophysiological mechanisms of cough. Moreover this observation may also provide 
a new way of investigating both normal and abnormal cough sounds, enabling clinically useful information to be extracted from the cough signal.

\section{References}

1. Evans JN, Jaeger MJ. Mechanical aspects of coughing. Pneumologie 1975; 152: 253-257.

2. Wynder EL, Lemon FR, Mantel N. Epidemiology of persistent cough. Am Rev Respir Dis 1964; 91: 679-699.

3. Loudon RG. Smoking and cough frequency. Am Rev Respir Dis 1976, 14: 1033-1036.

4. Collier JG, Fuller RW. Capsaicin inhalation in man and the effects of sodium cromoglycate. Br J Pharmacol 1984; 81: 113-117.

5. Loudon RG, Romans WE. Cough monitoring equipment. Med Res Eng 1967; 6: 25-30.

6. Korpas J, Sadlonova J, Salat D, Masarova E. The origin of cough sounds. Bull Eur Physiopathol Respir 1987; 23 (Suppl.): 47s-50s.

7. Piirila P, Sovijarvi ARA. Differences in acoustic and dynamic characteristics of spontaneous cough in pulmonary diseases. Chest 1989; 96: 46-53.

8. Stoneman SAT, Parker P, Jones A. Correlation of breath sounds with diagnosis of lung dysfunction: experimental observations. Proc Inst Mech Eng 1989; 203: 151-158.

9. Fuller RW, Choudry NB. Increased cough reflex associated with angiotensin converting enzyme inhibitor cough. Br Med J 1987; 295: 1025-1026.

10. Press WH, Flannery BP, Teukolsky SA, Vetterling WT. In: Numerical recipes in C. Cambridge University Press, 1988.

11. Kelemen SA, Cseri T, Marozsan I. Information obtained from tussigrams and the possibilities of their application in medical practice. Bull Eur Physiopathol Respir 1987; 23 (Suppl. 10): S51-S56.

12. Debreczeni LA, Korpas J, Salat D. Spectral analysis of cough sounds recorded with and without a noseclip. Bull Eur Physiopathol Respir 1987; 23: S57-S61.

13. Toop LJ, Thorpe CW, Fright R. Cough sound analysis: a new tool for the diagnosis of asthma? Fam Pract 1989; 6: 83-85.

14. Fuller RW, Karlsson JA, Choudry NB, Pride NB. Effect of inhaled and systemic opiates on responses to inhaled capsaicin in humans. J Appl Physiol 1988; 65(3): 11251130.

15. Karlsson JA, Sant'Ambrogia G, Widdicombe J. Afferent neural pathways in cough and reflex bronchoconstriction. J Appl Physiol 1988; 65: 1007-1023.

16. Maggi CA, Meli A. The sensory-efferent function of capsaicin-sensitive sensory neurons. Gen Pharmacol 1988, 19: 1-43.

17. Morice A, Lowry R, Brown M, Higgenbottom T. Tachyphylaxis and the cough reflex. Thorax 1988; 43: 255P.

18. Fuller RW, Dixon CMS, Barnes PJ. Bronchoconstrictor response to inhaled capsaicin in humans. J Appl Physiol 1985; 58: 1080-1084.

19. Thorpe CW, Fright WR, Toop LJ, Dawson KP. A microcomputer-based interactive cough sound analysis system. Comput Meth Prog Biomed 1991; 36: 33-43.

20. Young S, Abdul-Sattar N, Caric D. Glottic closure and high flows are not essential for productive cough. Bull Eur Physiopathol Respir 1987; 23 (Suppl. 10): 11s-17s.

21. Thorpe CW, Topp LJ, Dawson KP. Towards a quantitative description of asthmatic cough sounds. Eur Respir J 1992; 5: 685-692.

22. Holmes JN. In: Speech Synthesis and Recognition. Van Nostrand Reinhold (UK) Co. Ltd, 1988. 